\title{
IMMUNO-MAGNETIC BEADS ELISA FOR DIAGNOSIS OF SCHISTOSOMA MANSONI INFECTION
}

\author{
By
}

EMAN MOSTAFA H. MÉABED and ESSAM A. HASSAN

Department of Parasitology, and Department of Tropical Medicine, Faculty of

Medicine, Fayoum University, El Fayoum Postal Code 63513, Egypt

( ${ }^{\star}$ Correspondence:emeabed@gmail.com/emm02@fayoum.edu.eg)

\section{Abstract}

Diagnosis of Schistosoma mansoni infection is a challenge in low transmission regions. Serological diagnosis proved more sensitive and extensively used to detect anti- Schistosoma antibodies. This study evaluated the immunomagnetic beads-enzyme-linked immunosorbent assay (IMB-ELISA) for diagnosing $S$. mansoni infection in comparison with the commercial IHA, indirect ELISA and microscopy stool examination.

A total of 100 subjects were divided into four groups ( 25 each). G1: cases actively passing $S$. mansoni eggs, G2: chronic cases of S. mansoni, G3: cases with other parasitosis and G4: normal healthy subjects.

S. mansoni diagnostic rate was $25 \%, 46 \%, 48 \%$, \& $50 \%$ by parasitological, IHA, Indirect ELISA \& IMB-ELISA, respectively. In G1, the four tests gave positivity rates of $100 \%, 88 \%, 96 \%, \& 100 \%$, respectively. In $\mathrm{G} 2$, the four tests gave $0 \%, 84 \%, 88 \% \& 96 \%$, respectively. Sensitivity of various tests were estimated in relation to total true positive $S$. mansoni cases (G1 \& G2), and gave $50 \%, 86 \%$, $92 \%$ \& $98 \%$ for the applied tests, respectively. Cross-reactivity rates were estimated in relation to $S$. mansoni negative cases (G3 \& G4). Specificity rates were $100 \%, 94 \%, 96 \% \& 98 \%$, respectively.

Key words: Fayoum University Hospitals, Schistosoma mansoni, Magnetic beads, ELISA, IHA

\section{Introduction}

Schistosomiasis endemicity in both tropical and subtropical countries forms great health troubles, with around 200 million people infected and 700 million people at infection risk worldwide (WHO, 2012). WHO (2016) estimated that treatment must be provided for at least 206.4 million people, and only 89 million people of them were reported as treated. The WHO control plan depends on large-scale periodic population treatment to decrease the disease transmission, which necessitates prior diagnostic screening (WHO, 2018). In addition, sensitive and specific diagnostic tests are needed for early detection of cases prior development of any complications (WHO, 2018).

Microscopic egg detection, especially by Kato-Katz (KK) is the standard method for quantitative detection of $S$. mansoni eggs in fecal samples due to its high specificity (WHO, 2018). The test, however, is labourintensive, time-consuming, and to some extent messy due to low worm burden and/or high day to day fluctuation in egg counts. In addition, the test should be repeated to in- crease its sensitivity which raises its cost, and the laboratory hazards of handling stool samples.

For the regions where there is low disease transmission, follow up of the treated subjects necessitates using highly sensitive diagnostic techniques to replace copro-scopic searching for eggs which is a waste of time and efforts. False-negative subjects will act to maintain the disease endemicity, despite the application of control measures (Espirito-Santo et al, 2014; WHO, 2018).

PCR was utilized for schistosomiasis detection using various test forms. However, the method is not standardized and its sensitivity needs to be confirmed, in addition to the high cost needed for the equipment, chemicals and training the personnel exceeding the budget of primary health-care settings (Xu et al, 2011).

Immunological techniques for antibody detection have been widely used since they attracted the scientists' attention by their advantages over stool examination as being highly sensitive and easy for use. These included forms of indirect immunofluorescent- 
antibody tests (IFATs), IHAs, and ELISAs. Different antigenic preparations were applied such as the cercarial antigen (CA), the soluble egg antigen (SEA), and the purified or the crude adult worm antigen (AWA) (Kinkel et al, 2012). Several types of ELISA techniques have been successfully employed for the large-scale laboratory diagnosis of $S$. mansoni infection by using the antibodies against different types of antigens in automated and quantitative assays (EspiritoSanto et al, 2014).

The magnetic bead-based immunoassay was launched in 1980. It is commonly used to isolate nucleic acids, proteins, organelles, cells and other molecules. It was applied for hormones, cytokines and tumour markers detection (Liu et al, 2010). The magnetic separation was used in many forms in both indirect ELISA and Sandwich ELISA for detection of many parasitic infections as $S$. mansoni (Teixeira et al, 2007), S. japonicum (Liu et al, 2010; Lei et al, 2011; Xu et al, 2011; Yu et al, 2012a, b; 2014), toxoplasmosis (Hegazy et al, 2015), neurocysticercosis (Hernández-González et al, 2017).

This study aimed to evaluate the IMBELISA diagnosing $S$. mansoni infection in comparison with the IgG antibody-based detection by both IHA, and indirect ELISA and with the microscopy stool examination.

\section{Material and Methods}

This case-control study was conducted in the period from October 2015 to June 2017. The study received approval by the Local Health Authorities and the Ethics Committee of Faculty of Medicine, Fayoum University. Subjects were informed about the study aim and gave written consents.

Tested subjects: Subjects were 100: 72 males and 28 females, their age ranged from 12- 45 years. They were divided into four groups. G1: Active S. mansoni group: 25 cases with positive $S$. mansoni eggs in stool. Egg count by KK ranged from 50-100 egg/gram (EPG). They were negative for other parasites including S. haematobium. They were collected from rural health care units and the Center for Diagnosis of Schistosomiasis El-Hadkka (Fayoum Governorate). G2: Chronic S. mansoni group: 25 cases with a history of past infection and previous Praziquantel treatment and presented with symptoms and signs of chronic S. mansoni infection, and parasitologically negative for both types of schistosomiasis and other parasitic infections. G3: Cross-reactivity group: 25 cases positive for other parasitic infections; fascioliasis $(\mathrm{n}=4)$, ancylostomiasis $(n=9)$, and giardiasis $(n=12)$, and parasito-logically negative for both types of schistosomiasis. Cases of G2 \& G3 were chosen from the attendee of the Department of Tropical Medicine, Faculty of Medici- ne, Fayoum University. G4: negative con- trol group: 25 subjects with no history of previous exposure to canal water, and parasitologically negative for both types of schistosomiasis and other helminthes. They were selected from hospital attendee of matched age and sex as cases.

Sampling, Parasitological examination, and IHA: From each participant, two stool samples were taken on 2 consecutive days also a sample of $3 \mathrm{~mL}$ venous blood was withdrawn. Samples were delivered to the Department of Parasitology, Faculty of Medicine, Fayoum University, and sera were separated then stored at $-20^{\circ} \mathrm{C}$ until needed. Direct wet mount smear, and iodine-stained smear, Formol-éther concentration technique (Cheesbrough, 1987), and KK stool technique were performed to diagnose parasitic infections as 3 slides/stool sample (Katz et al, 1972).

The IHA was applied to detect anti-Schistosoma IgG antibodies (Fumouze Diagnostics, SOFIBEL, S.A.S., France) according to the manufacturer's directions. The red blood cells (RBCs) sensitized with Schistosoma antigens were added to serially diluted serum samples, and each sample was tested twice. In each test, non-sensitized RBCs, positive and negative control sera were included as controls. After $2 \mathrm{hr}$ incubation at room temperature, the dilution before that 
gave a clear sharp dark spot was recorded as the titer at a cutoff 1:160, and was presented as a reciprocal value.

Schistosoma mansoni egg antigen preparation: S. mansoni eggs were purchased from Theodor Bilharz Research Institute, Egypt. Further immunological tests were developed and adjusted in Parasitology Department, Faculty of Veterinary Medicine, Cairo University. S. mansoni egg antigen (SEA) was prepared by placing eggs in $0.9 \% \mathrm{NaCl}$, homogenization for $1 \mathrm{hr}$ on ice and 20,000 rpm centrifugation for $3 \mathrm{hr}$ at $4{ }^{\circ} \mathrm{C}$ (van Gool et $a l, 2002)$. Supernatant was collected, aliquoted, kept at $-20^{\circ} \mathrm{C}$, and the antigenic protein content was determined (Lowry et al, 1951).

Preparation of hyper-immune sera (RHIS) in rabbits: SEA was used to raise specific polyclonal antibodies in rabbits to be used as reference control sera in ELISA tests (Dunne et al, 1986). Rabbits were injected subcutaneously with $2.4 \mu \mathrm{g}$ of SEA emulsified in mineral oil. Three following injections were done using $1.2 \mu \mathrm{g}$ SEA divided into 3 doses at 2 weeks interval. Sera were collected of the vaccinated animals one week after the last injection. The collected sera were stored at $-20^{\circ} \mathrm{C}$ until used.

Coupling of $S$. mansoni SEA to magnetic beads: The magnetic beads-carboxy-terminated with diameter around $2 \mathrm{~mm}$ were brought (Beijing Bio Biology Company, Beijing, China) used according to $\mathrm{Yu}$ et al. (2012). At first, the beads were activated by adding them to $0.1 \mathrm{M}$ 2-morpholine-ethane- sulfonic acid hydrate (MES hydrate) buffer of pH 6.0, N-hydroxysuccinimide and 1-ethyl3-(dimethylaminopropyl)-carbodiimide hydrochloride (Sigma-Aldrich, St. Louis, Mo,

USA).The suspension was mixed and incubated for 20 minutes at $25^{\circ} \mathrm{C}$. This was followed by three times washing of the beads using $0.01 \mathrm{M}$ phosphate-buffered saline (PBS), pH 7.4. For antigenic coating, the $S$. mansoni SEA was added, mixed and incubated with the active beads at $37^{\circ} \mathrm{C}$ for 2 hours followed by two times washing steps. Bovine serum albumin (BSA-Sigma-Aldrich
St. Louis, Mo, USA), $1.5 \%$ (w/v) was added after the $2^{\text {nd }}$ beads-antigen incubation, for blocking of any free binding sites on the beads. The washing buffer (Tris-buffer solution with $0.05 \%$ Tween $20, \mathrm{pH}$ 7.4-TBST) was used to wash the beads (three times). The coated beads were added to TBST containing $0.01 \%$ sodium azide, BSA and kept at $4{ }^{\circ} \mathrm{C}$ until used.

Immunomagnetic beads-ELISA Technique: Specific IgG antibodies to $S$. mansoni SEA antigens present in sera were measured using IMB-ELISA (Yu et al, 2012), after checkerboard titration. The method was performed in small flat-bottom tubes fitted to a magnet rack (Lei et al, 2011). A $60 \mu \mathrm{L}$ of antigen-conjugated magnetic beads $(0.2 \mathrm{mg})$ were used per tube, and $30 \mu \mathrm{L}$ of serum sample diluted 1:200 in 0.01 M PBS ( $\mathrm{pH} 7.4$ ) was added to each tube. RHIS used as a reference positive control, negative control sera and PBS (blank control) were included in each test set. The entire mixture was incubated with continuous rotation for 20 minutes at $37^{\circ} \mathrm{C}$ to avoid IMB precipitation.

For washing, the first step was IMB separation. The tubes were placed for 2 minutes on the magnetic rack to remove the supernatant. The second step was adding the TBST to IMB with gentle vortexing to get rid of any unbound antibodies (repeated thrice). Then, $100 \mu \mathrm{L}$ of Horse-radish peroxidaseconjugated (HRPC) goat anti-human IgG and anti-rabbit IgG (Sigma, St. Louis, Mo, USA) diluted 1:1000 was added to the IMB, mixed for one hour at $37^{\circ} \mathrm{C}$, and then IMB were separated, the liquid was removed, and three times washing were performed as described before. After washing, $100 \mu \mathrm{L}$ of substrate buffer Ortho-phenylenediamidine (OPD) was added at a concentration of 340 $\mu \mathrm{g} / \mathrm{mL}$ and incubated with gentle shaking for 20 minutes at $37^{\circ} \mathrm{C}$. The reaction was terminated by addition of $300 \mu \mathrm{L}$ of stopping reagent $0.2 \mathrm{M} \mathrm{H}_{2} \mathrm{SO}_{4}$. IMBs were separated; the reaction fluid was transmitted to the polystyrene high binding flat-bottom 96-well ELISA plates (Libro, USA). Optical density 
(OD) was estimated using an automated Titerteckmultiscan plate reader (Titertek-Berthold, Berthold Detection Systems GmbH, Pforzheim, Germany), at 450nm (Lei et al, 2011). Serum samples were tested in triplicates and the mean OD was used as the final result. Cut-off values for assays were at least two times higher than mean OD for negative control group.

Indirect ELISA was performed (Doenhoff et al, 1993) after checkerboard titration. Overnight coating of the flat-bottom 96-well ELISA plates was performed using $200 \mu /$ well of $20 \mu \mathrm{g} / \mathrm{mL}$ of $S$. mansoni ESA in carbonate buffer ( $\mathrm{pH} 9.6$ ) at $4^{\circ} \mathrm{C}$.

The plates were washed with PBS $(50 \mathrm{mM}$, $\mathrm{pH} 7.2$ ), and blocked with $0.2 \%$ BSA in PBS Tween-20 (PBS-T, pH 7.2), 100 $\mu \mathrm{L} /$ well. After three times of washing, sera $(1: 100)$ were added $100 \mu \mathrm{L} /$ well, (three replicate/ sample), incubated for $2 \mathrm{hrs}$ at $37^{\circ} \mathrm{C}$. RHIS was used as a reference positive control. HRPC-goat anti-human and anti-rabbit IgG (1:2000) were pipetted, incubated at $37^{\circ} \mathrm{C}$ for $1 \mathrm{hr}$, followed by washing. Then, the substrate buffer, OPD, $340 \mu \mathrm{g} / \mathrm{mL}$ was added, kept for 15 minutes at room temperature, and terminated using $0.2 \mathrm{M} \mathrm{H}_{2} \mathrm{SO}_{4}$. The absorbance was read using the same ELISA plate reader at $450 \mathrm{~nm}$ as before.

Statistical analysis: Data were computerized and analyzed by SPSS 17 program (SPSS Inc, Chicago, Ill). Sensitivity (SN) and specificity (SP) rates of various tests were estimated. The degree of agreement (DA) between results of various tests was determined by Cohen's Kappa (K) index at $95 \%$ confidence intervals. The $\mathrm{K}$ index was reported as a poor DA if value $<0.20$, a low DA if ranged between $0.20 \& 0.40$, a moderate DA between $0.41 \& 0.60$, a good DA between $0.61 \& 0.80$, with an excellent DA between $0.8 \& 1.00$ (Altman, 1990). Bivariate or Pearson's correlation estimated the correlation between every two tests, and degree of significance. A $P$ value $\leq 0.01$ was considered significant. The receiver-operating characteristic (ROC) curve graphically plotted the test SN against (1- SP) of the reference standard technique. The cut-off value gave the best balance of SN \& SP, and area under the curve (AUC) determined the test degree of accuracy.

\section{Results}

For the egg passers group (G1), parasitological examination, IHA, Indirect ELISA and IMB-ELISA tests detected 100\%, $88 \%$, $96 \%, \& 100 \%$ of cases, respectively. For $S$. mansoni chronic cases (G2) the four tests detected $0 \%, 84 \%, 88 \% \& 96 \%$, respectively. The tests $\mathrm{SN}$ rates were estimated in relation to total number of true positive $S$. mansoni cases (G1 \& G2), and gave 50\%, $86 \%, 92 \% \& 98 \%$ for the four tests, respectively.

For cross-reactivity group (G3) the four tests detected $0,3,2 \& 1$ fascioliasis cases, respectively. No cross-reactivity was detected with sera of cases infected with other parasites or normal control sera. Cross-reactivity or SP rate was estimated in relation to S. mansoni negative sera (G3 \&G4). The SP rates were $100 \%, 94 \%, 96 \% \& 98 \%$, respectively.

Out of 100 subjects, 25 cases were positive by parasitological tests, while IHA, Indirect ELISA and IMB-ELISA tests detected $46 \%$, $48 \%$, \& $50 \%$ of cases, respectively. Mean \pm standard deviation (SD) of optical density (OD) values (OD) of positive and negative samples detected by the indirect ELISA \& IMB-ELISA tests given (Tab.1).

Application of tests on $S$. mansoni infected groups (G1, G2) and the estimated $\mathrm{SN}$ rates of different tests in comparison with IMBELISA were presented (Table 2). For the group actively passing eggs (G1), SN of parasitological examination, indirect ELISA and IHA tests were $100 \%, 96 \% \& 88 \%$, respectively. SN rates for chronic cases (G2) were $0 \%, 91.7 \%$ and $87.5 \%$ respectively. Total SN rates in both $S$. mansoni groups (G1, G2) were $51 \%, 93.9 \%$ and $87.8 \%$, respectively. 
Table 1 . Sensitivity and specificity rates detected by different techniques at different groups

\begin{tabular}{|c|c|c|c|c|c|c|}
\hline \multicolumn{3}{|c|}{ Group (number) } & $\begin{array}{l}\text { Parasitology } \\
\text { No. + ve (\%) }\end{array}$ & $\begin{array}{l}\text { IHA } \\
\text { No. +ve(\%) }\end{array}$ & $\begin{array}{l}\text { Indirect ELISA } \\
\text { No.+ve }(\%)\end{array}$ & $\begin{array}{l}\text { IMB-ELISA } \\
\text { No. +ve(\%) }\end{array}$ \\
\hline \multicolumn{3}{|c|}{ G1 (25) } & $25(100)$ & $22(88)$ & $24(96)$ & $25(100)$ \\
\hline \multicolumn{3}{|c|}{ G2 (25) } & $0(0.0)$ & $21(84)$ & $22(88)$ & $24(96)$ \\
\hline \multicolumn{3}{|c|}{ Sensitivity \% } & 50 & 86 & 92 & 98 \\
\hline \multirow[t]{4}{*}{ G3 } & \multicolumn{2}{|c|}{ Fascioliasis (4) } & $0(0.0)$ & $3(75)$ & $2(50)$ & $1(25)$ \\
\hline & \multicolumn{2}{|c|}{ Ancylostomiasis (9) } & $0(0.0)$ & $0(0.0)$ & $0(0.0)$ & $0(0.0)$ \\
\hline & \multicolumn{2}{|c|}{ Giardiasis (12) } & $0(0.0)$ & $0(0.0)$ & $0(0.0)$ & $0(0.0)$ \\
\hline & \multicolumn{2}{|c|}{ Total G3 (25) } & $0(0.0)$ & $3(12)$ & $2(8)$ & $1(4)$ \\
\hline \multicolumn{3}{|c|}{ G4 (25) } & $0(0.0)$ & $0(0.0)$ & $0(0.0)$ & $0(0.0)$ \\
\hline \multicolumn{3}{|c|}{ Specificity \% } & 100 & 94 & 96 & 98 \\
\hline \multicolumn{3}{|c|}{ Total detection rate } & $25(25)$ & $46(46)$ & $48(48)$ & $50(50)$ \\
\hline \multirow{2}{*}{\multicolumn{2}{|c|}{ OD range }} & Positive & - & - & $0.32-0.763$ & $0.5-1.9$ \\
\hline & & Negative & - & - & $0.05-0.015$ & $0.1-0.2$ \\
\hline \multicolumn{3}{|c|}{$\mathrm{M} \pm \mathrm{SD}$ positive $\mathrm{OD}$ values } & - & - & $0.65 \pm 0.25$ & $1.8 \pm 0.25$ \\
\hline \multicolumn{3}{|c|}{ Cut off value } & - & 160 & 0.222 & 0.5 \\
\hline
\end{tabular}

Table 2: Sensitivity rates of various tests in relation to IMB-ELISA for diagnosis of $S$. mansoni groups.

\begin{tabular}{|c|c|c|c|c|c|c|c|c|c|c|c|c|c|}
\hline \multirow{2}{*}{$\begin{array}{l}\text { Tested } \\
\text { group }\end{array}$} & \multirow[t]{2}{*}{ IMB-ELISA } & \multicolumn{3}{|c|}{ Parasitology } & \multicolumn{3}{|c|}{ ELISA } & \multicolumn{3}{|c|}{ IHA } & \multicolumn{3}{|l|}{$\mathrm{SN} \%$} \\
\hline & & $\mathrm{P}$ & $\mathrm{N}$ & $\mathrm{T}$ & $\mathrm{P}$ & $\mathrm{N}$ & $\mathrm{T}$ & $\mathrm{P}$ & $\mathrm{N}$ & $\mathrm{T}$ & Parasitology & ELISA & IHA \\
\hline \multirow{3}{*}{$\begin{array}{l}\text { G1: active } \\
\text { S. mansoni }\end{array}$} & Positive (P) & 25 & 0 & 25 & 24 & 1 & 25 & 22 & 3 & 25 & \multirow[t]{3}{*}{100} & \multirow[t]{3}{*}{$96 \%$} & \multirow[t]{3}{*}{88} \\
\hline & Negative(N) & 0 & 0 & 0 & 0 & 0 & 0 & 0 & 0 & 0 & & & \\
\hline & Total $(\mathrm{T})$ & 25 & 0 & 25 & 24 & 1 & 25 & 22 & 3 & 25 & & & \\
\hline \multirow{3}{*}{$\begin{array}{l}\text { G2: chron- } \\
\text { ic } S . \text { man- } \\
\text { soni }\end{array}$} & $\mathrm{P}$ & 0 & 24 & 24 & 22 & 2 & 24 & 21 & 3 & 24 & \multirow[t]{3}{*}{0} & \multirow[t]{3}{*}{91.7} & \multirow[t]{3}{*}{87.5} \\
\hline & $\mathrm{N}$ & 0 & 1 & 1 & 0 & 1 & 1 & 0 & 1 & 1 & & & \\
\hline & $\mathrm{T}$ & 0 & 25 & 25 & 22 & 3 & 25 & 21 & 4 & 25 & & & \\
\hline \multirow[t]{3}{*}{ Total } & $\mathrm{P}$ & 25 & 24 & 49 & 46 & 3 & 49 & 43 & 6 & 49 & \multirow[t]{3}{*}{51} & \multirow[t]{3}{*}{93.9} & \multirow[t]{3}{*}{87.8} \\
\hline & $\mathrm{N}$ & 0 & 1 & 1 & 0 & 1 & 1 & 0 & 1 & 1 & & & \\
\hline & $\mathrm{T}$ & 25 & 25 & 50 & 46 & 4 & 50 & 43 & 7 & 50 & & & \\
\hline
\end{tabular}

The 100 subjects were compared regarding SN and SP rates (Tab. 3). In comparison with microscopy examination, estima-ted $\mathrm{SN}$ rates increased gradually from IHA $(88 \%)$ to Indirect ELISA (96\%) then IMBELISA tests $(100 \%)$, while SP rates were $68.0 \%, 68.0 \% \& 66.7 \%$, for the three tests, respectively. ROC curve analysis for comparison of various tests with parasitological examination was built and presented with its data (Fig. 1, Tab. 4).

In comparison with IMB-ELISA test, the performance of other tests was presented (Tab. 3). Parasitological examination, IHA and indirect ELISA test $\mathrm{SN}$ rates were $50.0 \%, 88.0 \%$ \& $94.0 \%$, respectively, while SP rates were $100 \%, 96 \%$ and $98 \%$, respectively.

In comparison with indirect ELISA, sensitivity rates of microscopy examination, IHA
\& IMB-ELISA tests were $50 \%, 93.8 \%$ \& $97.9 \%$, respectively, but specificity were $98.1 \%, 98.1 \% \& 94.2 \%$, resp-

ectively.

In comparison with IHA test, sensitivity rates of microscopy examination, IMBELISA \& indirect ELISA test were $47.8 \%$, $95.7 \%$, \&97.8\%, respectively, but specificity rates were $94.4 \%, 88.9 \%$ \& $94.4 \%$, respectively.

Kappa index for degrees of agreement (DA) and $P$ value estimated form the bivariate correlation between results of each two tests given (Tab. 3). DA was excellent between every two serological tests ( $\mathrm{K}$ index $>$ 0.8 ), with a significant correlation between them $(\mathrm{P} \leq 0.001)$. There were moderate $\mathrm{DA}$ between microscopy examination and each of three serological assays ( $\mathrm{K}$ index ranged from 0.437- 0.5). 
Table 3: Comparison of sensitivity (SN) and specificity (SP) rates, degree of concordance (Kappa index), and $P$ value from bivariate correlation between results of every two tests used to diagnose $S$. mansoni infection among total examined subjects (n 100)

\begin{tabular}{|c|c|c|c|c|c|c|c|c|c|c|c|c|c|}
\hline \multirow{2}{*}{\multicolumn{2}{|c|}{ Test }} & \multicolumn{3}{|c|}{$\begin{array}{l}\text { Parasitological } \\
\text { examination }\end{array}$} & \multirow[t]{2}{*}{$\begin{array}{l}{ }^{\mathrm{d}} \mathrm{SN} \& \mathrm{SP} \text { comp- } \\
\text { ared to parasitology }\end{array}$} & \multicolumn{3}{|c|}{ IHA } & \multirow[t]{2}{*}{$\begin{array}{l}{ }^{\mathrm{c}} \mathrm{SN} \& \mathrm{SP} \text { co- } \\
\text { mpared to IHA }\end{array}$} & \multicolumn{3}{|c|}{ Indirect ELISA } & \multirow{2}{*}{$\begin{array}{l}{ }^{b} \text { SN \& SP com- } \\
\text { pared to indirect } \\
\text { ELISA }\end{array}$} \\
\hline & & $\mathrm{P}$ & $\mathrm{N}$ & $\mathrm{T}$ & & $\mathrm{P}$ & $\mathrm{N}$ & $\mathrm{T}$ & & $\mathrm{P}$ & $\mathrm{N}$ & $\mathrm{T}$ & \\
\hline \multirow{4}{*}{$\begin{array}{l}\text { a IMB-ELISA } \\
\text { data } \\
\text { and SN and } \\
\text { SP rates } \\
\text { compared to } \\
\text { IMB-ELISA }\end{array}$} & $\mathrm{P}$ & 25 & 25 & 50 & \multirow{4}{*}{$\begin{array}{l}{ }^{d} \mathrm{SN}=100 \% \\
\mathrm{SP}=66.7 \%\end{array}$} & 44 & 6 & 50 & \multirow{4}{*}{$\begin{array}{l}{ }^{\mathrm{c}} \mathrm{SN}=95.7 \\
\% \\
\mathrm{SP}=88.9 \%\end{array}$} & 47 & 3 & 50 & \multirow{3}{*}{$\begin{array}{l}{ }^{b} \mathrm{SN}=97.9 \% \\
\mathrm{SP}=94.2 \%\end{array}$} \\
\hline & $\mathrm{N}$ & 0 & 50 & 50 & & 2 & 48 & 50 & & 1 & 49 & 50 & \\
\hline & $\mathrm{T}$ & 25 & 75 & 100 & & 46 & 54 & 100 & & 48 & 52 & 100 & \\
\hline & & \multicolumn{3}{|c|}{$\begin{array}{l}{ }^{\mathrm{a}} \mathrm{SN}=50.0 \% \\
\mathrm{SP}=100 \% \\
\mathrm{~K}=0.5\end{array}$} & & \multicolumn{3}{|c|}{$\begin{array}{l}{ }^{\mathrm{a}} \mathrm{SN}=88.0 \% \\
\mathrm{SP}=96.0 \% \\
\mathrm{~K}=0.84 \\
\mathrm{P} \leq 0.001\end{array}$} & & \multicolumn{3}{|c|}{$\begin{array}{l}{ }^{a} \mathrm{SN}=94.0 \% \\
\mathrm{SP}=98.0 \% \\
\mathrm{~K}=0.92 \\
\mathrm{P} \leq 0.001\end{array}$} & \\
\hline \multirow{4}{*}{$\begin{array}{l}{ }^{6} \text { Indirect } \\
\text { ELISA data } \\
\text { and SN and } \\
\text { SP rates } \\
\text { compared to } \\
\text { indirect } \\
\text { ELISA }\end{array}$} & $\mathrm{P}$ & 24 & 24 & 48 & \multirow{3}{*}{$\begin{array}{l}{ }^{\mathrm{d}} \mathrm{SN}=96 \% \\
\mathrm{SP}=68.0 \%\end{array}$} & 45 & 3 & 48 & \multirow{3}{*}{$\begin{array}{l}{ }^{c} \mathrm{SN}=97.8 \% \\
\mathrm{SP}=94.4 \%\end{array}$} & & & & \\
\hline & $\mathrm{N}$ & 1 & 51 & 52 & & 1 & 51 & 52 & & & & & \\
\hline & $\mathrm{T}$ & 25 & 75 & 100 & & 46 & 54 & 100 & & & & & \\
\hline & & \multicolumn{3}{|c|}{$\begin{array}{l}{ }^{\mathrm{b}} \mathrm{SN}=50.0 \% \\
\mathrm{SP}=98.1 \% \\
\mathrm{~K}=0.49\end{array}$} & & \multicolumn{3}{|c|}{$\begin{array}{l}{ }^{\mathrm{b}} \mathrm{SN}=93.8 \% \\
\mathrm{SP}=98.1 \% \\
\mathrm{~K}=0.92 \\
\mathrm{P} \leq 0.001 \backslash\end{array}$} & & & & & \\
\hline \multirow{4}{*}{$\begin{array}{l}\text { IHA, SN } \\
\text { \&S rates of } \\
\text { Parasitology } \\
\text { compared to } \\
\text { IHA }\end{array}$} & $\mathrm{P}$ & 22 & 24 & 46 & \multirow{3}{*}{$\begin{array}{l}{ }^{d} \mathrm{SN}=88 \% \\
\mathrm{SP}=68.0 \%\end{array}$} & & & & & & & & \\
\hline & $\mathrm{N}$ & 3 & 51 & 54 & & & & & & & & & \\
\hline & $\mathrm{T}$ & 25 & 75 & 100 & & & & & & & & & \\
\hline & & $\begin{array}{l}{ }^{c} \mathrm{~S} \\
\mathrm{SP} \\
\mathrm{K}=\end{array}$ & $\begin{array}{l}=47 \\
94.4 \\
.437\end{array}$ & & & & & & & & & & \\
\hline
\end{tabular}

${ }^{\mathrm{a}}$ Performance of various tests in relation to IMB-ELISA, ${ }^{\mathrm{b}}$ Performance of various tests in relation to indirect ELISA. ${ }^{\mathrm{c}}$ Performance of various tests in relation to IHA, ${ }^{\mathrm{d}}$ Performance of various tests in relation to parasitological examination

Table 4: ROC Curve data comparing the performance of various tests to standard microscopy

\begin{tabular}{|l|c|c|c|c|c|}
\hline \multirow{2}{*}{ Test used } & \multirow{2}{*}{ Area } & \multirow{2}{*}{ Std. Error } & \multirow{2}{*}{ Asymptotic Sig. } & \multicolumn{2}{|c|}{ Asymptotic 95\% Confidence Interval } \\
\cline { 5 - 6 } & & & & Lower Bound & Upper Bound \\
\hline Indirect-ELISA_ & 0.914 & 0.029 & 0.000 & 0.858 & 0.970 \\
\hline IMB_ELISA & 1.000 & 0.000 & 0.000 & 0.000 & 1.000 \\
\hline IHA & 0.780 & 0.051 & 0.000 & 0.680 & 0.880 \\
\hline
\end{tabular}

\section{Discussion}

Application of governmental control programs including snail control and provision of Praziquantel therapy has decreased schistosomiasis transmission in Egypt. This created a low level of transmission and infection intensity, where microscopy failed to detect cases. This required highly sensitive and specific serological tests to detect infected cases before spreading infection and development of complications (Kinkel et al, 2012; Hinz et al, 2017). These tests were needed for accurate follow up of cases after treatment to indicate antibody titres, in egg negative cases with high antibody positivity indicating re-infection or treatment failure (Yu et al, 2012).

In low transmission regions of schistosomiasis, antigen-based detection assays using monoclonal antibodies including circulating antigens neither showed high SN \& SP for detection of chronic cases nor better than antibody-based detection to assess the chemotherapy outcome (Gryseels et al, 2006;
Legesse and Erko; Zhu et al, 2012). The IMB-ELISA for antibody detection was effective with the same sensitivity as antigen detection and evading costs to prepare monoclonal antibodies. The test was indicated in areas with low transmission levels (Liu et al, 2010).

The present study compared three antibody-based tests for diagnosis of schistosomiasis mansoni including IMB-ELISA.

The results showed that the IMB-ELISA had high SN and SP, with minimal crossreactivity rates with other parasites, while indirect-ELISA and IHA showed different levels of cross-reactivity with cases of fascioliasis. Although ELISA showed high SN in detecting egg-passers, the SN to confirm chronic cases was lower than IMB-ELISA. The IMB-ELISA detection rate for chronic schistosomiasis was $96 \%$, with significant differences from the results by using the indirect-ELISA (88\%) and IHA (84\%).

The present results of indirect ELISA ranged with previous studies. Van Gool et al 
(2002) reported SN \& SP rates of $93.3 \%$ \& $98.8 \%$ for indirect SEA-ELISA to detect $S$. mansoni egg passers. Kinkle et al (2012) compared three commercial indirect ELISA and three home-made indirect ELISA tests using three different antigens (AWA, SEA, $\&$ cercarial antigens) together with IHA to diagnose S. mansoni egg-passers, the SN range of applied ELISA tests was (47.6$90.5 \%)$ and the SP was $(88.4-100 \%)$. The indirect SEA-ELISA test achieved SN \& SP rates of $90.5 \% \& 97.1 \%$, respectively (Kinkleet al, 2012). As regard S. mansoni infection, the indirect SEA-ELISA test had a SN rate ranged from $56 \%-100 \%$ and SP ranged from 6\%-99\%, respectively (Hinz et al, 2017).

The present results were compared with $\mathrm{Yu}$ et al (2012) using IMB-SEA ELISA versus indirect ELISA for diagnosing S. japonicum infection in persons with low-intensity infection. The IMB-SEA ELISA had a higher SN of $96.55 \%$ than indirect ELISA $91.38 \%$ with significant difference $(\mathrm{P}<$ $0.01)$. In follow up of treated cases, SN of IMB-SEA ELISA was $73.3 \%$, but ELISA was $66.7 \%(\mathrm{P}<0.01)$. The two tests performed similarly when tested for crossreactivity gave a specificity of $50 \%$. Also, the magnetic micro-bead separation to detect $S$. japonicum antibodies showed that magnetic ELISA method was more precise and sensitive than traditional SEA-ELISA in the epidemiological survey (Liu et al, 2010).

The present results of IHA test were in range with previous studies that evaluated its performance for serodiagnosis of schistosomiasis. It gave SN of $88 \%$ \& SP of $98.9 \%$ for $S$. mansoni egg-passers (van Gool et al, 2002). Kinkle et al. (2012) estimated SN \& SP rates of $76.2 \% \& 99 \%$ for IHA tests for $S$. mansoni egg-passers. In comparison with the microscopy, the SN \& SP of magnetic ELISA were $99 \% \& 80.9 \%$, while the SN \& SP of IHA were $62.9 \%$ and $84.5 \%$ detecting S. japonicum antibodies (Liu et al, 2010).

By comparing the 4 types of IHA tests with indirect ELISA and magnetic beads FITC
ELISA with the standard KK technique, SN of IHAs ranged from 92\%-98\%, indirect ELISA was $95 \%$, \& FITC ELISA was $98 \%$. The SP of IHAs ranged from 70\%-93\%, indirect ELISA was $93.6 \%$, and FITC ELISA was $94.3 \%$ (Xu et al, 2011).

In the present study, the high SN \& SP for IMB-ELISA was explained by using the magnetic beads as the solid phase in the immunoassay. The magnetic beads increased the surface area for antigen or antibody binding helped by gravity. So, they were easy with quick and complete separation of antigen or antibody from supernatant solution without loss of reactants in the washing buffer, and reduced time required for coating or incubation step from $12-14 \mathrm{hr}$ to $2 \mathrm{hr}$ (Liu et al, 2010). The technique rendered completely automated, and it's safe, decreasing the manual labour and provision of rapid, precise results. It requires simple methods and could replace the indirect ELISA \& IHA (Liu et al, 2010; Yu et al, 2012). Also, the test can be used for antigen detection with very high SN \& SP in Sandwich ELISA technique after the availability of the specific monoclonal antibodies.

\section{Conclusion}

IMB-ELISA proved to be a sensitive and specific method for diagnosis of $S$. mansoni in patients with low-intensity infections.

\section{References}

Altman, DG, 1990: Practical Statistics for Medical Research (Chapman \& Hall/CRC Te-xts in Statistical Science) $1^{\text {st }}$ Edition. London, UK.

Cheesbrough, M, 1987: Medical Laboratory Manualfor Tropical Countries, Anatomy, Physiology, Clinical Chemistry and Parasitology. $2^{\text {nd }}$ edition, Butterworth-Heinemann.

Doenhoff, MJ, Butterworth, AE, Hayes, R J, et al, 1993: Seroepidemiology and serodia- gnosis of schistosomiasis in Kenya using cru-de and purified egg antigens of Schistosoma mansoni in ELISA. Trans. R. Soc. Trop. Med. Hyg. 87:42-8. Dunne, DW, Agnew, AM, Modha, J, Doenhoff, MJ, 1986: Schistosoma mansoni egg antigens: Preparation of rabbit antisera with monospecific immune-precipitating activity, \& their use in antigen characterization. Parasite Immunol.8, 6:575-86. 
Espírito-Santo, MC, Alvarado-Mora, MV, Dias-Neto E, et al, 2014: Evaluation of realtime PCR assay to detect Schistosoma mansoni infections in a low endemic setting. BMC Infect. Dis. 14:55-8.

Gryseels, B, Polman, K, Clerinx, J, Keste- ns, L, 2006: Human schistosomiasis. Lancet 368 : 1106-18.

Hegazy, S, Farid, A, Rabae, I, El-Amir, A, 2015: Novel IMB-ELISA assay for rapid diagnosis of human toxoplasmosis using SAG1 Antigen. Jpn. J. Infect. Dis. 68, 6:474-80.

Hernández-González, A, Noh, J, Perteguer, M J, Gárate, T, Handali, S, 2017: Comparison of T24H-his, GST-T24H \& GST-Ts8B2 recombinant antigens in western blot, ELISA and multiplex bead-based assay for diagnosis of neurocysticercosis. Parasit. Vectors 10, 1:23-7.

Hinz, R, Schwarz, NG, Hahn, A, Frickmann, H, 2017: Serological approaches for the diagnosis of schistosomiasis: A review. Mol. Cell Probes 31:2-21.

Katz, N, Chaves, A, Pellegrino, J, 1972: A Simple device for quantitative stool thick smear technique in Schistosoma mansoni. Rev. Inst. Med. Trop. São Paulo 14, 6:397-400.

Kinkel, HF, Dittrich, S, Bäumer, B, Weitzel, T, 2012: Evaluation of eight serological tests for diagnosis of imported schistosomiasis. Clin. Vacci. Immunol. 19, 6:948-53.

Legesse, M, Erko, B, 2008: Field-based evaluation of a reagent strip test for diagnosis of schistosomiasis mansoni by detecting circulating cathodic antigen (CCA) in urine in low endemic area in Ethiopia. Parasite 15:151-5.

Lei, J, Su, B, Xu, H, et al, 2011: Evaluation of an IgY-based immunomagnetic enzyme-linked immunosorbent assay system for detection of circulating Schistosoma japonicum antigen in serum samples from patients in China. Am. J. Trop. Med. Hyg. 85, 6:1054-9.

Liu, Z, Zhang, L, Yang, H, Zhu, Y, Jin, W, et al, 2010: Magnetic microbead-based enzymelinked immunoassay for detection of Schistosoma japonicum antibody in human se-rum. Anal. Biochem. 15, 4042:127-34.
Lowry, OH, Rosenbrough, NJ, Farr, AL, Randall, RJ, 1951: Protein measurement with folin-phenol reagent. J. Biol. Chem. 193, 1: 26575.

Teixeira, CF, Neuhauss, E, Ben, R, et al, 2007: Detection of Schistosoma mansoni eggs in feces through their interaction with paramagnetic beads in a magnetic field. PLoSNegl. Trop. Dis. 1:e73.

Van Gool, T, Vetter, H, Vervoort, T, et al, 2002: Serodiagnosis of imported schistosomiasis by a combination of a commercial indirect hemagglutination test with Schistosoma mansoni adult worm antigens and an enzyme-linked immunosorbent assay with $S$. mansoni egg antigens. J. Clin. Microbiol. 40, 9:3432-7.

WHO, 2012: Schistosomiasis: Fact sheet $n^{\circ}$ 115, Geneva, Switzerland.

WHO, 2018: Schistosomiasis: Fact sheet, Geneva, Switzerland.

Xu, J, Peeling, RW, Chen, JX, et al, 2011: Evaluation of immunoassays for the diagnosis of Schistosoma japonicum infection using archived sera. PLoS Negl. Trop. Dis. 5, 1:e949.

Yu, Q, Yang, H, Feng, Y, Zhu, Y, Yang, X, 2012a: Magnetic affinity enzyme-linked immuno-assay for diagnosis of schistosomiasis japonicum in persons with low-intensity Infection. Am. J. Trop. Med. Hyg. 87, 4:689-93.

Yu, Q, Yang, H, Feng, Y, Yang, X, Zhu, Y, 2012b: Magnetic affinity enzyme-linked immuno-assay based on recombinant $26 \mathrm{kDa}$ glutathione-S-transferase for serological diagnosis of schistosomiasis japonica. Acta Trop. 124, 3:199202.

Yu, Q, Yang, H, Guan, F, Feng, Y, Yang, $X$, et al, 2014: Detection of IgG in sera of patients with schistosomiasis japonica by developing magnetic affinity enzyme-linked immunoassay based on recombinant 14-3-3 protein. Trans. R. Soc. Trop. Med. Hyg. 108, 1:37-41.

Zhu, Y, Hua, W, Xu. M, et al, 2012: A novel immunodiagnostic assay to detect serum antibody response against selected soluble egg antigen fractions from Schistosomajaponicum. PLoS One 7, 8:e44032. 


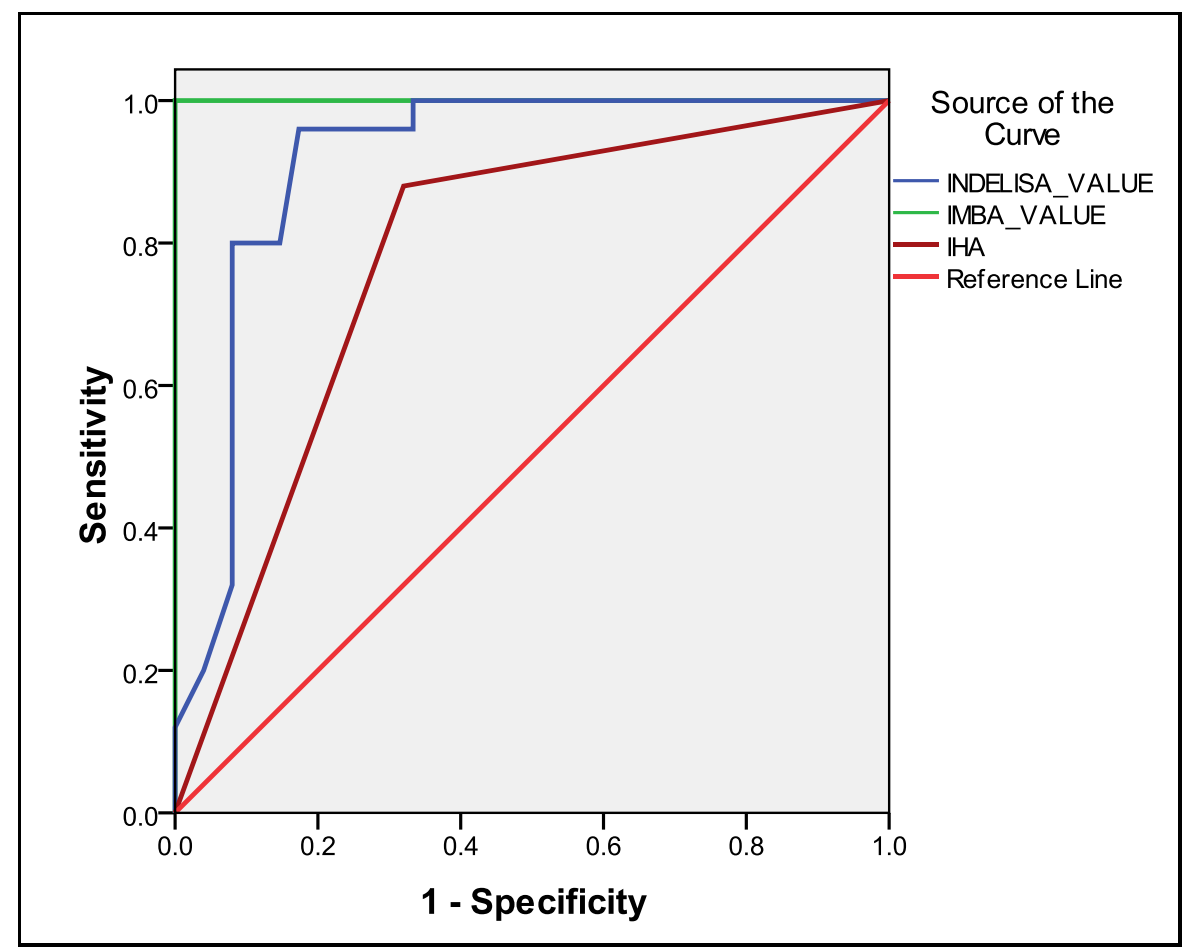

